Apuntes Universitarios, 2021: 11(3), julio-setiembre ISSN: 2304-0335 DOI: https://doi.org/10.17162/au.v11i3.690

\title{
Comunicación terapéutica del profesional de enfermería y donación de órganos
}

\section{Therapeutic communication of nursing and organ donation}

\author{
Ana María Amancio Castro, ${ }^{1 a}$ Zobeida Elizabet Camarena Vargas, ${ }^{2}$ María del Pilar Fajardo \\ Canaval, ${ }^{3}$ y Sofía Del Carpio Flórez ${ }^{4}$ \\ Universidad Inca Garcilaso de la Vega. Lima, Perú ${ }^{1234}$ \\ iD Orcid ID: https://orcid.org/0000-0001-5227-6752 ${ }^{1}$ \\ (iD) Orcid ID: https://orcid.org/0000-0003-2053-4803² \\ (iD) Orcid ID: https://orcid.org/0000-0001-9942-04913 \\ (iD) Orcid ID: https://orcid.org/0000-0003-3513-8782
}

Recibido: 19 de octubre 2020

Aceptado: 20 de marzo de 2021

\begin{abstract}
Resumen
A lo largo de la civilización, la donación de un órgano es considerado un gesto altruista, el acto de mayor bondad entre los seres humanos. Por ello, la comunicación terapéutica es una herramienta útil para conducir este proceso. En ese sentido, el objetivo de este estudio es determinar la relación entre la comunicación terapéutica del profesional de enfermería y la donación de órganos. Se trata de un estudio de enfoque cuantitativo, de tipo correlacional, de corte transversal, para el cual se recolectó la información utilizando un cuestionario de 47 ítems, los primeros 25 miden la variable comunicación en situaciones críticas, y las siguientes 22 miden la variable actitud hacia la donación de órganos aplicado a 119 profesionales de enfermería. El análisis inferencial demostró que existe una relación directa entre la comunicación terapéutica del profesional de Enfermería y la donación de órganos, con un valor de 0.701, significativo según la prueba estadística de la Rho de Spearman concluyentes, es decir, existe un nivel de comunicación de regular a buena. Se pudo observar una relación directa y positiva entre la comunicación terapéutica y la donación de órganos.
\end{abstract}

Palabras claves: Donación de órganos, comunicación terapéutica, profesional de enfermería.

\begin{abstract}
Throughout civilization organ donation is an altruistic gesture taking into account as the major act of kindness between human beings and therapeutic communication is a useful tool to contribute in this process. The objective tried to determine the relations hip between therapeutic communication of registered nurses and organ donation. Materials and Methods: This study has a quantitative
\end{abstract}


approach; information was collected using a questionnaire that was applied to 119 registered nurses. Results: denotative results $50.4 \%$ and communicative goals $54,62 \%$ are conclusive that it exists a good level of communication from regular to good. Discussion: Results comparable with a study made by Romero, Y., who found that it exists an attitude of negative direction that would contribute to decrease probability of donation, harmful to people requiring transplant, intensity is adverse. Verifying concordances with obtained data in this research because they denote medium level in both variables with direct and positive relationship. Conclusions: It was observed a direct and positive relationship between therapeutic communication and organ donation.

Key words: Organ donation, therapeutic communication, nursing profesional

\section{Introducción}

La comunicación en situaciones críticas utilizada a diario por todos los profesionales de la salud, es considerada como una situación que genera tensión en la atención del paciente y su familia. Los profesionales expertos en cuidados paliativos han definido la comunicación de malas noticias como un acto humano, ético, médico y legal. Atero (2017) refiere que es muy doloroso brindar información al paciente o familiar sobre su situación de salud, peor aún informarles que su ser querido se encuentra muy cercano al deceso.

La teoría de la comunicación terapéutica sustentada en la comunicación de la enfermera con el paciente y/o familia, permite la práctica de habilidades blandas y técnicas, capaces de enfrentar los retos que se le presente y es definida como la relación de ayuda mutua, formando un nuevo enfoque de los cuidados que brinda el licenciado en enfermería donde el centro del trabajo es el paciente (Inio, 2015).

Considerando que el cuidado es el quehacer diario del licenciado en enfermería que requiere desarrollar habilidades comunicacionales que transfieran la confianza necesaria para la identificación oportuna de sus necesidades sociales, emocionales y espirituales; por tanto, consideramos la imperiosa necesidad de desarrollar las habilidades de atención, escucha activa y diálogo hacia el paciente (Fernández, 2016).

La comunicación terapéutica del licenciado en enfermería debe darse a nivel denotativo, utilizando términos o palabras de manera clara veraz y objetiva (Gonzales, 2019) las mismas que facilitaran la identificación de signos y explicación de sus peculiaridades (Bautista, Arias y Carreño, 2016). Dentro de esta perspectiva, a nivel meta comunicativo referido a la comunicación en la que se utilizan gestos, actitudes interpersonales y emociones; depende de la escucha activa 
directamente la aceptación de la donación de órganos (García, 2018); la empatía, actitud con la cual es posible identificarse con otra persona, la falta de ello conlleva al desapego, la incomprensión, el desinterés y consecuentemente, afecta de manera directa los sentimientos de toda persona (Triana, 2017).

Desde la perspectiva más general, en la donación de órganos se está refiriendo a tres tipos de donaciones: la donación en vida, la que se refiere a los familiares o las amistades del receptor que de forma desinteresada otorgan un órgano al paciente; la donación post mortem, la que se da cuando el donante ha fallecido, y la donación de personas próximo al deceso o en asistolia (Gómez, Ballena y León, 2016). Considerando lo descrito líneas arriba, la donación de órganos es un procedimiento complejo que implica riesgo, que está predispuesto a las circunstancias en las que se emite una información, así como al ritmo y la aceptación que cada individuo tiene sobre la vida o la muerte (Sáez y Aranda, 2017).

En el Perú, según el diario El Peruano (2019), existían 6972 pacientes que están incluidos en la lista de espera para trasplante de órganos y tejidos, ese mismo año la Dirección de Donantes y Trasplantes del Ministerio de Salud (MINSA), reporto en los últimos tres años, que la proporción de donantes de órganos por cada millón de habitantes en el Perú ha crecido de 1.9 donantes a 2.3 donantes por millón de peruanos (Romero, 2015).

Según la Organización Nacional de Donación y Trasplante (ONDT), en ese mismo año fueron detectados 1223 posibles donantes, de los cuales 275 fueron donantes potenciales y 62 donantes efectivos de órganos y tejidos, es decir tasa de 2 donantes por millón de habitantes, datos que demuestran la trascendencia de donar y por ende la importancia del este estudio.

Ahora bien, la comunicación terapéutica de la enfermera, con el paciente y familia juega un papel importante en el acto de donar, ya que, en el gran número de casos no apoyan la decisión del donador, los dolientes muestran negativa, debido al impacto emocional, este les imposibilita procesar la información que están recibiendo y, como consecuencia, aumenta la negación al proceso (Zamora y Diaz, 2018). Sobre la base de todo lo descrito anteriormente, se desarrolló la presente investigación cuyo objetivo fue determinar la relación entre la comunicación terapéutica del profesional de enfermería y la donación de órganos. 


\section{Materiales y métodos}

El presente estudio fue desarrollado con un enfoque cuantitativo, utilizando el método hipotético-deductivo, que se origina desde la verdad general hasta el abordaje del conocimiento particular o específico. En cuanto al diseño que se utilizó en la presente investigación, fue el no experimental transversal. Fonseca et al. (2013), manifiesta que son diseños en los que el investigador no se limita solo a recoger los datos observados de las variables en la realidad.

El muestreo fue de tipo aleatorio simple conformada por una población de 119 profesionales de Enfermería, como unico criterio de inclusión se determino ser profesionales de enfermería de áreas criticas del hospital en estudio durante el segundo semestre 2019, a quienes se les solicitó por escrito el consentimiento de participación voluntaria, los datos se recolectaron utilizando el cuestionario de "Comunicación del profesional de enfermería y actitud para donación de órganos” un solo instrumento, divididos en dos partes, 25 items midieron la variable comunicación en situaciones críticas y 22 items la variable actitud hacia la donación de organos, diseñado y elaborado por los autores, con criterios de pertinencia, relevancia y claridad; la validez se realizo a traves de 5 jueces expertos con cuyas respuestas se aplicó la V de Aiken con un resultado del (0.88 y 0.79, p $0.001<0.05)$. La confiabilidad se obtuvo con el estadístico alfa de Cronbach, aplicado en una prueba piloto.

Con la información recolectada, se elaboró la base Excel, buscando obtener la sumatoria de los valores de las dimensiones y de las variables, información que fue procesada con el programa SPSS versión 25.0, facilitando el convertir las variables a niveles - rangos, consecuentemente a ello, se elaboró el informe.

Todos los corolarios finales se realizaron de manera agrupada y ordenada, considerando estos de ayuda para describir, así como observar la posible relación de las variables de la investigación. Finalmente, la prueba de hipótesis se realizó utilizando la estadística no paramétrico Rho de Spearman.

Por su parte, el proyecto fue aprobado por el Consejo Universitario de la Universidad Inca Garcilaso de la Vega, con código A-2019-INVEST-02-01, y por el Comité de Docencia e Investigación del Hospital Santa Rosa, código 19/044-HSR-OADI, categorizado con riesgo bajo para los participantes. Se garantizó a los colaboradores resguardar el anonimato de la información, por lo que aceptaron ser parte de la muestra de manera consciente, firmando el consentimiento informado. Se les notificó la posibilidad de la publicación de los resultados del estudio. 


\section{Resultados}

\section{Análisis descriptivo}

En la tabla 1, se muestra la distribución de los datos demográficos donde se observa: en cuanto a la edad, que la mayoría, un 39.50 \%, presenta edades entre 20 a 34 años; en el caso del sexo, la mayoría, un 77.31 \%, son del sexo femenino; por otro lado, en el tema de la religión, la mayoría son católicos y cristianos, con un porcentaje del 57.98 \%; en cuanto al estado civil, la mayoría, un 37.82 \% son casadas; en razón del cargo, la mayoría de la muestra son enfermeras asistenciales, un 76.47 \%, y, finalmente, en el punto de la procedencia, la mayoría son de la sierra y la costa.

\section{Tabla 1}

Datos demográficos

\begin{tabular}{|c|c|c|c|c|c|}
\hline Edad & N. ${ }^{\circ}$ & $\%$ & Estado civil & N. ${ }^{\circ}$ & $\%$ \\
\hline (20-34 años) & 47 & 39.50 & Soltera & 20 & 16.81 \\
\hline (35-44 años) & 44 & 36.97 & Conviviente & 22 & 18.49 \\
\hline (45-66 años) & 28 & 23.53 & Casada & 45 & 37.82 \\
\hline Sexo & $\mathbf{N}$ & $\%$ & Divorciada & 22 & 18.49 \\
\hline Femenino & 92 & 77.31 & Viuda & 10 & 8.40 \\
\hline Masculino & 27 & 22.69 & Cargo & N. ${ }^{\circ}$ & $\%$ \\
\hline Religión & N. ${ }^{\circ}$ & $\%$ & $\begin{array}{l}\text { Enfermera } \\
\text { asistencial }\end{array}$ & 91 & 76.47 \\
\hline Católica & 43 & 36.13 & $\begin{array}{l}\text { Jefa de } \\
\text { servicio }\end{array}$ & 17 & 14.29 \\
\hline Cristiana & 26 & 21.85 & Supervisora & 11 & 9.24 \\
\hline Adventista & 12 & 10.08 & Procedencia & N. ${ }^{o}$ & $\%$ \\
\hline Evangelista & 26 & 21.85 & Costa & 51 & 42.86 \\
\hline Ateo & 7 & 5.88 & Sierra & 47 & 39.50 \\
\hline \multirow[t]{2}{*}{ Otro } & 5 & 4.20 & Selva & 21 & 17.65 \\
\hline & & & Total & 119 & 100.00 \\
\hline
\end{tabular}

Fuente: Datos obtenidos por los investigadores

En la tabla 2, se muestra la distribución de los datos concluyentes de la comunicación terapéutica en sus dimensiones denotativa, con un resultado de $50.4 \%$, y metacomunicativa, con un resultado de $54.62 \%$. Ambas revelan un resultado regular. 


\section{Tabla 2}

Dimensiones de la comunicación terapéutica

\begin{tabular}{lllllll}
\hline & $\begin{array}{l}\text { Dimensión } \\
\text { denotativa }\end{array}$ & & \multicolumn{2}{l}{$\begin{array}{l}\text { Dimensión } \\
\text { metacomunicativa }\end{array}$} & $\begin{array}{l}\text { Variable Comunicación } \\
\text { terapéutica }\end{array}$ \\
\hline Niveles & F & \% & F & \% & F & \% \\
Bueno & 31 & 26.05 & 25 & 21.01 & 28 & 23.53 \\
Regular & 60 & 50.42 & 65 & 54.62 & 62 & 52.10 \\
Malo & 28 & 23.53 & 29 & 24.37 & 29 & 24.37 \\
Total & 119 & 100.00 & 119 & 100.00 & 119 & 100.00 \\
\hline
\end{tabular}

Fuente: Datos obtenidos por el investigador

En la tabla 3, en los datos obtenidos referentes a la donación de órganos, se aprecia un $52.94 \%$ de nivel medio, un $15.13 \%$ en nivel alto y el $31.93 \%$ percibe un nivel bajo.

\section{Tabla 3}

Distribución de la donación de órganos

\begin{tabular}{lll}
\hline Organización & $\mathrm{F}$ & $\%$ \\
\hline Alto & 18 & 15.13 \\
Medio & 63 & 52.94 \\
Bajo & 38 & 31.93 \\
Total & 119 & 100.00 \\
\hline
\end{tabular}

Fuente: Datos obtenidos por los investigadores

En la tabla 4, Se analiza los datos de las dimensiones de la donación de órganos el resultado más relevante en un nivel medio a la dimensión post mortem, en un 58.33 \%, y la donación próxima al deceso o en asistolia, en un $31.93 \%$. 


\section{Tabla 4}

Distribución comparativa de las dimensiones de la donación de órganos

Donación en vida $\quad$ Donación post mortem $\begin{gathered}\text { Donación de personas próximo al } \\ \text { deceso o en asistolia }\end{gathered} \quad$ Total $\quad \%$

\begin{tabular}{lcccccccc}
\hline Organización & $\mathrm{f}$ & $\%$ & $\mathrm{~F}$ & $\%$ & $\mathrm{f}$ & $\%$ & & \\
Alto & 2 & 13.33 & 9 & 15.00 & 7 & 15.91 & 18 & 15.13 \\
Medio & 5 & 33.33 & 35 & 58.33 & 23 & 52.27 & 63 & 52.94 \\
Bajo & 8 & 53.33 & 16 & 26.67 & 14 & 31.82 & 38 & 31.93 \\
Total & 15 & 100.00 & 60 & 100.00 & 44 & 100.00 & 119 & 100.00 \\
\hline
\end{tabular}

Fuente: Datos obtenidos por los investigadores.

El análisis inferencial demostró que existe una relación directa entre la comunicación terapéutica del profesional de Enfermería y la donación de órganos, con un valor de 0.701, significativo según la prueba estadística de la Rho de Spearman, el mismo que indica relación directa entre las variables del estudio. Tablas No 4 y 5

\section{Tabla 5}

Prueba de correlación entre la comunicación terapéutica del profesional de enfermería y donación de órganos

\begin{tabular}{|c|c|c|c|c|}
\hline & & & $\begin{array}{l}\text { Comunicación } \\
\text { terapéutica }\end{array}$ & $\begin{array}{l}\text { Donación de } \\
\text { órganos }\end{array}$ \\
\hline Rho & de & Coeficiente & de 1,000 &, $701^{* *}$ \\
\hline \multirow{3}{*}{ Spearman } & Comunicació & correlación & & \\
\hline & n terapéutica & Sig. (bilateral) & . & ,001 \\
\hline & & $\mathrm{N}$ & 119 & 119 \\
\hline
\end{tabular}




\section{Tabla 6}

Cruce de información

\begin{tabular}{|c|c|c|c|c|}
\hline \multirow[t]{2}{*}{ Variable } & \multicolumn{3}{|c|}{ Donación de órganos } & \multirow[t]{2}{*}{ Valor p } \\
\hline & Alto & Medio & Bajo & \\
\hline & (18) & $(63)$ & (38) & 0.001 \\
\hline $\begin{array}{l}\text { Edad (Media } \pm \text { de o } \\
\text { mediana y RI) }\end{array}$ & 47 (20-34 años) & $\begin{array}{l}44 \text { (35-44 } \\
\text { años) }\end{array}$ & $\begin{array}{l}28 \text { (45-66 } \\
\text { años) }\end{array}$ & $\begin{array}{c}\text { Mediana } \\
\text { (35) }\end{array}$ \\
\hline Genero \%(n) & $\%(n)$ & $\%(n)$ & $\%(n)$ & Total \\
\hline Femenino & $12.60 \%(15)$ & $40.33 \%(48)$ & $\begin{array}{l}24.37 \\
\%(29)\end{array}$ & $\begin{array}{c}77.31 \% \\
(92)\end{array}$ \\
\hline Masculino & $2.52 \%(3)$ & $12.60 \%(15)$ & $7.56 \%(9)$ & $\begin{array}{c}22.68 \% \\
(27)\end{array}$ \\
\hline \multicolumn{5}{|c|}{ Variable Comunicación terapéutica } \\
\hline Buena & 7 & 12 & 9 & $\begin{array}{c}23.53 \% \\
(28)\end{array}$ \\
\hline Regular & 7 & 31 & 24 & $\begin{array}{c}52.10 \% \\
(62)\end{array}$ \\
\hline Mala & 4 & 20 & 5 & $\begin{array}{c}24.37 \% \\
\text { (29) }\end{array}$ \\
\hline \multicolumn{5}{|l|}{ Dimensión denotativa } \\
\hline Buena & 8 & 14 & 9 & $\begin{array}{c}26.05 \% \\
(31)\end{array}$ \\
\hline Regular & 6 & 30 & 24 & $\begin{array}{c}50.42 \% \\
(60)\end{array}$ \\
\hline Mala & 4 & 19 & 5 & $\begin{array}{c}23.53 \% \\
(28)\end{array}$ \\
\hline \multicolumn{5}{|c|}{ Dimensión metacomunicativa } \\
\hline Buena & 7 & 10 & 8 & $\begin{array}{c}21.01 \% \\
(25)\end{array}$ \\
\hline Regular & 7 & 31 & 27 & $\begin{array}{c}54.62 \% \\
(65)\end{array}$ \\
\hline Mala & 4 & 22 & 3 & $\begin{array}{c}24.37 \% \\
\text { (29) }\end{array}$ \\
\hline
\end{tabular}

Fuente: Datos obtenidos por los investigadores 


\section{Discusión}

La estrategia necesaria para una comunicación efectiva es trabajar sobre la escucha activa, exteriorizar la empatia que sentimos hacia el usuario, indagar sobre sus preocupaciones o dudas, se requiere entonces el uso de aquellas herramientas que permitira al profesional enfermero establecer una comunicación efectiva, es decir saber utilizar la comunicación de acuerdo al tipo de paciente; asi como evaluar su accionar para estar segura de lo que esta transmitiendo (Lopez, 2018).

La comunicación terapéutica es pieza clave en el cuidado de enfermería, a traves de la cual se puede conocer la exigencias del paciente, no solo las que exterioriza verbalmente, sino las que intentra comunicarnos a traves de su mensaje con las expresiones no verbales como los gestos corporales y el contacto ocular (Espinoza , 2017).

Aunque los profesionales médicos son pieza clave en la atención a los paciente basicamente como responsables de brindar información sobre el estado y evolución de su salud; son los licenciados en enfermería responsables de los cuidados, los que mantienen una relación más precisa con el paciente y familia; haciendo impresindible las demostraciones de empatía, afecto y cuidado humano que se brinda (Marvan, Orihuela, y Alvares, 2020).

Solo así estaremos preparados para brindar información real a la población buscando sensibilizarlas al dolor de los otros, dimitiendo fabulas, perjucios, miedos y desconfianzas que existe en torno a una donación, y mas bien fortalecer las capacidades de ayuda al projimo para contribuir a la mejora de la calidad de vida (Espinoza, Zapata, y Mejia, 2017).

En el estudio realizado encontramos que el profesional de enfermería en la atención diaria al paciente utiliza la comunicación denotativa como la metacomunicación, uno en mayor porcentaje que el otro; tal como lo manifiestan. Pino, Murillo, y Suárez, (2019) refieren que la utilización de ambos tipos de comunicación son complementarias, durante la entrevista con los usuarios (paciente/familia), considerando en este proceso la situación de salud que están viviendo los usuarios suele determinar o condicionarlos al tomar una decisión tan importante y trascendente como es el acto de donador algún órgano o tejido, para ello requerimos la participación de todos y todas.

Como se puede observar en los resultados, el 77,31\% de la población son mujeres, en edades de 20 a 34 años (39,50\%). Montero et.al (2018) encontraron datos similares a los nuestro ellos hallaron 86,6\% es población femenina, con buenos conocimientos sobre el proceso de donación. 
Marvan, Orihuela, y Alvares (2020) ponen enfasis en la importacia que se le otorga a la religión considera que las licenciadas en enfermería cree que la religión se opone la donación al morir, por lo que se le dificulta comunicarse con los pacientes o familiares, en una población de personal de medicina y de enfermería obtuvo como resultado mayor cantidad de catolicos (75.4 \%) y el (70;8\%) de estado civil casados; estos resultados se encuentran similares a los resultados presentados en esta investigación (36,13\%) de católicos así como (37,82\%) casados.

Sáez y Aranda (2017) refieren, que se requiere dominar habilidades comunicacionales en especial la escucha activa, que involucra el desarrollar capacidades cognitivas, empáticas, credibilidad, tono adecuado, demostración de elegir correctamente el momento y lugar para brindar comunicación; estrategias necesarias que aseguran una comunicación eficaz y efectiva con el paciente, en el proceso condicionado por la actitud de la persona, su nivel sociocultural, el contexto, sus experiencias y conocimiento sobre el tema. Manifestaciones, vinculadas a la necesidad desarrollar los sentidos, que les permita un nivel de percepción y sensibilidad, que le facilite estar conciente de lo que nos comunica y lo que es comunicado por el usuario, el enfermero es responsable del cuidado integral en aspectos biopsicosocial espiritual, requiere poner en practica la comunicación verbal y no verbal, buscando un nivel de aceptación y comprensión optima sobre el proceso de donar (Espinoza, 2017).

Becerra, et. al. (2019) concluyeron que la comunicación en la dimensión verbal es de carácter positivo, relevando la escucha activa (75\%), mientras que la comunicación no verbal (98,3\%).a predomio de la postura de “acercamiento” y “respeto” que muestran (98,3\%). estos resultados en relación a nuestro estudio son superiores, pues se encontro que el nivel predenominante en la dimensión denotativa (comunicación verbal) fue regular (50,42\%), con referencia a la comunicación no verbal (metacomunicación) se encontro resultado tambien de nivel regular $(52,10 \%)$.

Durante la comunicación terapeutica es importante mostrar sensibilidad al mirar y/o escuchar, expresión que brindan apoyo emocional y cuidados al individuo y familia. Estos aspectos son de dominio del profesional enfermero, dada su preparación eminentemente cientifica y en profundos valores éticos, morales, que hace de ella un ser sensible ante el dolor ajeno, aspecto que permite brindar cuidado, holistico, en aspectos biosipcosocial y espiritual (Triana, 2017).

Por su parte, Watson refiere que es necesario que el licenciado en enfermería haga buen uso de las herramientas que le permitan una comunicación verbal y no verbal efectiva para lograr un 
cuidado humanístico (Martinez, et. al.,2017). En el año 2019, de 324 potenciales donantes, 75 fueron donantes efectivos, obteniendo una tasa 2,3 donantes por millón de habitantes (Benavides, 2020). La utilización de los órganos para trasplantes en seres humanos se constituye en una disyuntiva ética y de enfrentamientos bioéticos especialmente en países occidentales, donde la primera barrera a la donación y trasplante de órganos se encuentra en las creencias religiosas de los profesionales de salud, puestos que son ellos los que no acepta la donación post mortem (Laidouni, Briones, y Garrido, 2017).

Sobre las bases de las ideas expuestas, los resultados mostrados por Jasso et.al (2017) señalan un 73,5 \% de sujetos posee actitud favorable a la donación post morten, en comparación con los datos obtenidos en el estudio estos son superiores, puesto que se encontro una escala de aceptación entre los profesionales baja 53.33\% cuando se trata de donación en vida y media en el caso de la donación post mortem 58.33\%.

La posición social o la situación de salud no son condiciones que puedan determinar o condicionar a un ser humano para que acepte o decline de ser un donador potencial de algún órgano o tejido. Sin embargo, el sufrir de alguna patología se constituye en un impedimento irreversible que permite efectuarse algún tipo de donación.

La principal limitación presentadas está, relacionado a los sesgos de temporalidad, pues la muestra estuvo constituida por profesionales de tres hospitales, datos tomados en un sólo momento. Sin embargo, nos invita a desarrollar otros estudios de nivel metacéntrico que nos permitan evaluar mejor el panorama regional, nacional o local, estando clara la idea del performance de los enfermeros que puede permitir re direccionar algunas políticas en salud sobre donación de órganos y de fortalecimiento de las habilidades comunicativas del profesional enfermero.

La comunicación terapéutica del profesional de enfermería en la donación de órganos, debe ser competente, puesto que es el personal idoneo de proporcionar a los familiares las información que permita el posicionamiento ante la contingencia de la donación; al respecto Conesa, et al (2004) refieren que la población española en general opina favorablemente ante la aceptación de donar un órgano; sin embargo, esta actitud inicial tan favorable sólo es efectiva en 77 \% de los posibles donantes, para disminuir esta diferencia se requiere proceder directamente en la comunidad a través de una adecuada información que facilite un estado sólido de opinión favorable a la donación. Por su parte, Alves, et al (2020) en el estudio realizados en Brasil encontró 
que del total de artículos examinados el 60\% se destaca el engrandecimiento del rol de la enfermera; pues aseveran que en la de donación de órganos el proceso comunicacional del profesional es piedra angular, cimentada en lo humano de las relaciones, producto de la convivencia sostenida entre los licenciados en enfermería y los familiares ante el eminente deceso de su paciente. En esta perspectiva, el estudio evidencia resultados finales que prueban una relación lineal estadística significativa, entre la comunicación terapeutica y la donación de órganos al nivel de 0,01 .

\section{Conclusiones}

Se concluye que la correlación entre comunicación terapéutica y las donaciones produjo un resultado significativo, en relación directa con la donación de órganos, $(\mathrm{Rho}=0.7)$, nivel de regular a media, no obstante es meritorio considerar el proceso de la comunicación terapéutica como una estrategia esencial para lograr el fortalecimiento de la cultura de donación en la población; mediante el análisis de los resultados se ha identificado que las habilidades del licenciado en enfermería en los niveles denotativo requieren ser fortalecidos, poniendo énfasis en aspectos técnicos, en el posicionamiento y el manejo de la situación durante la comunicación que debe sostener con el usuario del cual pretendemos acepte donar algún órganos.

En lo que respecta al nivel meta comunicativo, se requiere mejorar la preparación del profesional en aspectos relacionados a la empatía, la escucha activa, entre otros; lo que permitirá administrar cuidados de enfermería no diferenciados al paciente y familia, acciones que debe ser tomadas desde el nivel de pre grado.

\section{Referencias}

Achury, D. \& Pinillos, M. ( 2016). La comunicación con la familia del paciente que se encuentra al final de la vida. Enfermería Universitaria, 13(1), 55-60. doi:http://dx.doi.org/10.1016/j.reu.2015.12.001

Alves, C., Pergola-Marconato, A. \& Borges, M.. (2020). Equipo de enfermería en la donación de órganos. Revista Bioética 28(1), 76-82. doi:10.1590/1983-80422020281369 
Atero-López, D. (2017). Enfermería y la comunicación de malas noticias en el proceso de donación de órganos: revisión bibliográfica. Repositorio de Trabajos Académicos de la Universidad de Jaén. Obtenido de http://tauja.ujaen.es/jspui/handle/10953.1/6564

Bautista, L., Arias, M. \& Carreño, Z. (2016). Percepción de los familiares de pacientes criticos hospitalizados respecto a la comunicación y apoyo emocional. Revista Cuidarte 7(2), 1971309. doi:http://dx.doi.org/10.15649/cuidarte.v7i2.330

Benavides, M. (2020). Guía de procedimientos para el mantenimiento del donante potencial de órganos tejidoas humanos. Obtenido de file://C:/Users/Director/Desktop/RD\%20N\%C2\%B0\%20000124-2020-DGINSNSB\%20GP\%20008\%20Gu\%C3\%ADa\%20de\%20Procedimiento\%20para\%20el\%2 0Mantenimiento\%20del\%20Donante\%20Potencial.pdf

Beserra, G., Oliveira, P., Pagliuca, L., Almeida, P., Anjos, \& S Pinheiro, A. (2019). Comunicación no verbal entre enfermero y parturienta en trabajo de parto en países lusohablantes. Rev. Latino-Am. Enfermagem 27, 1-9. doi: http://dx.doi.org/10.1590/1518-8345.3032.3193.

Conesa, C., Zambudio, A., Ramírez, O., Rodríguez, M, Cotresas, K .\& Parrilla, P.(2004). Importancia de los profesionales de atención primaria en la educación sanitaria de la donación de órganos. Rev. Atención primaria 62, 528-535. doi:https://doi.org/10.1016/S0212-6567(04)70858-6

Espinoza, M. ( 2017). Comunicación que brinda la enfermera durante la hospitalización: Perspectiva de los familiares del paciente crítico - 2016. Tesis de maestría, Universidad Cesar Vallejo. Obtenido de http://repositorio.ucv.edu.pe/bitstream/handle/20.500.12692/21812/Espinoza_HMS.pdf?s equence $=1 \&$ is Allowed $=\mathrm{y}$

Espinoza, N., Zapata, C., \& Mejia, L. (abril de 2017). Conspiración de silencio: una barrera en la comunicación médico, paciente y familia. Rev Neuropsiquiatría, 80(2), 125-136. doi:http://dx.doi.org/https://doi.org/10.20453/rnp.v80i2.3105

Fernández, S. (2016). La comunicación terapéutica: acompañando a la persona en el camino de la enfermedad. Tribuna $\quad$ Panace, $111-114$. https://dialnet.unirioja.es/servlet/articulo?codigo=5794530 
Fonseca, A. (2013). Tipos de investigación. En A. Fonseca, B. Rojas, \& S. Martel, Investigación cientifica en salud con enfoque cuantitativo. Lima: Graficas S.R.I.R.L.

Garcia, J. (2009). Comunicación: la escucha activa. Obtenido de http://www.psicoterapeutas.com/index.html

Gómez, K., Ballena, J., \& León, F. (2016). Mitos sobre la donación de órganos en personal de salud, potenciales receptores y familiares de potenciales donantes en un hospital peruano: estudio cualitativo. Rev Peru Med Exp Salud Publica 33(1), 83-91. doi:https://doi.org/10.17843/rpmesp.2016.331.2011

Gonzales, P. (2019). Qué es el lenguaje connotativo y denotativo. Barcelona, España. Obtenido de https://www.unprofesor.com/lengua-espanola/que-es-el-lenguaje-connotativo-ydenotativo-con-ejemplos-2491.html

Inio, A. (2015). Comunicación terapéutica: Requisitos fundamentales para acompañar emocionalmente al paciente paliativo y su familia. Trabajo de fin de grado. Universitat Internacional de Catalunya. Obtenido de https://www.recercat.cat/bitstream/handle/2072/243032/Alessandro_Inio.pdf?sequence=1 : http://hdl.handle.net/2072/243032

Laidouni, N., Briones, E., \& Garrido, l. (2017). Percepción de los profesionales de la salud sobre las creencias y las tradiciones derivadas del Islam como barreras a la donación de órganos en Argelia. $\quad$ Gaceta Sanitaria 32(2), 123-131. doi:http://dx.doi.org/10.1016/j.gaceta.2016.09.012

López, R. (2018). El cuidado de la familia en el proceso de donación y trasplante de órganos. Tesis de fin de grado. Repositorio Universidad Autonoma de madrid. Obtenido de http://hdl.handle.net/10486/684902

Lopez, S. (2018). ¿Tienen los profesionales enfermeros formación en comunicación terapéutica? $\begin{array}{llll}\text { Enfermería día } & \text { al } & \text { 33-34. }\end{array}$ https://www.enfermeria21.com/revistas/metas/articulo/81026/actualidad-enfermeratienen-los-profesionales-enfermeros-formacion-en-comunicacion-terapeutica/

Martinez, M., Lopez, R., Reyes-Berlanga, M., Linares-Segovia, B., Martínez-Bernal, S., Ojeda, M., Reyes Gutierrez, K., Navarro, E., Colio, M., Rodriguez, S. (2017). Actitud hacia la 
donación de órganos en los principales hospitales generales del estado de Guanajuato. Revista Mexicana de Transplantes, 1, 6-11. https://www.medigraphic.com/cgibin/new/resumen.cgi?IDARTICULO=71414

Marvan, L., Orihuela, F., \& Alvares, A. (2020). Conocimientos generales y actitudes hacia la donación de órganos en una muestra de estudiantes mexicanos de medicina y de enfermería. Revista de Ciencias de la Salud, 18(2), 1-19. doi:https://doi.org/10.12804/revistas.urosario.edu.co/revsalud/a.9240

Millera, C., \& Breakwell, R. (2018). What factors influence a family’s decision to agree to organ donation? A critical literature review. London Jurnal of Primal Care, 10(4), 103-107. doi:10.1080/17571472.2018.1459226

Montero, A., Martinez, S., Fiera, E., Fernánez, A., Castro, D. \& Fernandez, D. (2018). Conocimientos y Actitudes ante la donación de órganos de los profesionales sanitarios de un Hospital de tercer Nivel. Esp Salud Pública, 92, doi:http://dx.doi.org/10.1016/j.resuscitation.2010.02.021

Pino, K., Murillo, M., \& Suárez, L. (2019). Acompañamiento al enfermo crónico o terminal y calidad de vida en familia. Poiésis 126-146. doi:https://doi.org/10.21501/16920945.3194

Polanco, F. (29 de abril de 2016). La comunicación en situaciones criticas. El Portal. Obtenido de https://www.elportal.com.do/alfredo-polanco/

Romero, Y. (2015). Actitud de los familiares hacia la donación de órganos en el servicio de cuidados intermedios en el Hospital Nacional Hipólito Unanue. Tranajo de Investigación. UNMSM. Obtenido de: https://cybertesis.unmsm.edu.pe/handle/20.500.12672/13252

Sáez, I., \& Aranda, B. (2017). Relación de ayuda de enfermería a pacientes y familiares en la unidad de cuidados intensivos. Revista Española de Comunicación en Salud 8(2), 239 246. doi:https://doi.org/10.20318/recs.2017.4004

Triana, M. (2017). La empatia en la relación enfermera-paciente. Avances en Enfermería, 35(2), 121-122. doi:http://dx.doi.org/10.15446/av.enferm.v35n2.66941 
Zamora, A., \& Diaz, Y. (2018). Factores que inciden en la cultura de donación de órganos en Morelia Michuacan. Revista de Ciencias Sociales, 12(44), 99-119. doi:orcid.org/00000003-1811-4711 